\title{
Motion analysis in video surveillance using edge detection techniques
}

\author{
Anupam Mukherjee ${ }^{1}$, Debaditya Kundu ${ }^{2}$ \\ ${ }^{1}$ (Department of Computer Science \& Engineering, Siliguri Institute of Technology, Siliguri, India) \\ ${ }^{2}$ (Department of Information Technology, Siliguri Institute of Technology, Siliguri, India)
}

\begin{abstract}
Motion tracking is an important task in image processing applications. To track moving objects and their interaction in a complex environment is a difficult task, this work basically explains the technique of tracking moving objects. Moving object detection can be accomplished by image capturing, background subtraction and Prewitt edge detection operator. The main idea of our approach, called the background subtraction technique, is to subtract directly between two consecutive frames to extract the difference image. The difference image marks the areas where a moving object was in frame $N$ and where the object is in frame $N+1$, respectively. Prewitt operator is more suitable in case of moving object analysis.
\end{abstract}

Keywords - Background subtraction, Canny edge detection operator, Edge detection, Motion Tracking, Moving object detection, Noise reduction, Prewitt edge detection operator.

\section{INTRODUCTION}

Edge detection is an important part of digital image processing, it is not only reduces the amount of data but also maintain useful structural information[1].In this paper we analyzed and visually compare between Canny and Prewitt edge detection operators.. This work is done by using following phases; In the first phase we focused on Edge detection followed by motion tracking with the help of background subtraction and in the last phase prewitt edge detected result is compared with Canny edge detected results. All of these operations are implemented by using Matlab2012a which is best suited for image processing applications. Moving object detection is a basis for a number of important applications, such as real-time surveillance and visual tracking [4]. In this paper we have proposed five different techniques for motion tracking, which will help us to track moving objects in a complex environment.

\section{EDGE DETECTION}

Edges are significant local changes of intensity in an image. Edges typically occur on the boundary between two different regions in an image. The goal of edge detection is to produce something like a line drawing of an image. In practice we will look for places in the image where the intensity changes quickly. Observe that, in general, the boundaries of objects tend to produce sudden changes in the image intensity[5]. For example, different objects are usually different colours or hues and this causes the image intensity to change as we move from one object to another ${ }^{[4]}$. In addition, different surfaces of an object receive different amounts of light, which again produces intensity changes ${ }^{[5]}$.

\section{TYPES OF EDGES}

Edges can be modeled according to their intensity profiles [2].

Step edge: the image intensity abruptly changes from one value to one side of the discontinuity to a different value on the opposite side.

Ramp edge: a step edge where the intensity change is not instantaneous but occur over a finite distance.

Roof edge: a ridge edge where the intensity change is not instantaneous but occur over a finite distance (generated usually by the intersection of surfaces).

\section{BACKGROUND SUBTRACTION}

Background subtraction is a technique in the fields of image processing and computer vision wherein an image's foreground is extracted for further processing (object recognition etc)[6]. Generally an image's regions of interest are objects (humans, cars, text etc) in its foreground. After the stage of image preprocessing (which may include image denoising etc) object localisation is required which may make use of this technique. Background subtraction is a widely used approach for detecting moving objects in videos from static cameras. The rationale in the approach is that of detecting the moving objects from the difference between the current frame and a reference frame, often called "background image", or "background model". Background subtraction is mostly done if the image is a part of a video stream. 


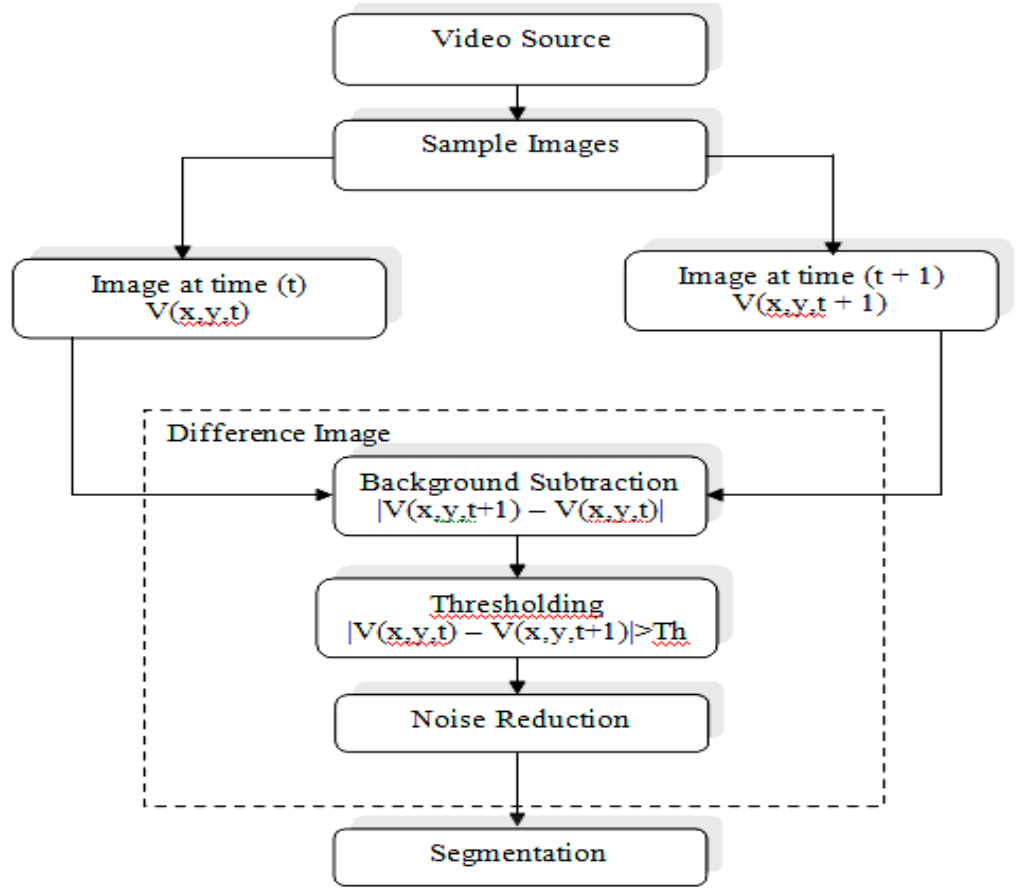

Figure1. Flow chart of Background Subtraction.

V.

Algorithms For Real Time Moving OBJECT DETECTION

Five different approaches for moving objection detection are described[4].

\section{V.I Motion Tracking: Using Single Prewitt Operator.}

Step1: Read Video Sequences by using Video Reader.

Step2: Capture two consecutive images and store it in IMG and IMG1.

Step3: $\mathrm{IMG:}=$ Convert Frame number $\mathrm{N}$ to Gray Scale.

Step4: IMG1: $=$ Convert Frame number $\mathrm{N}+1$ to Gray Scale.

Step5: Subtract frame $\mathrm{N}$ from frame $\mathrm{N}+1$, to generate the difference image.

Step6: Remove noise by using median filter.

Step7: Run Prewitt operator on the difference image in order to remove noise and to detect the edges.

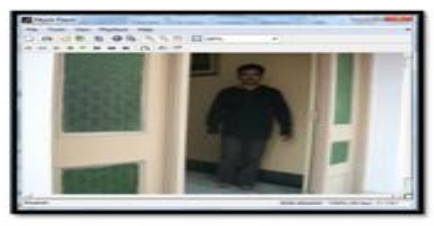

(a)

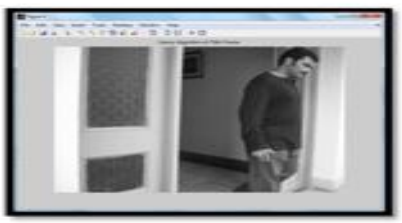

(c)

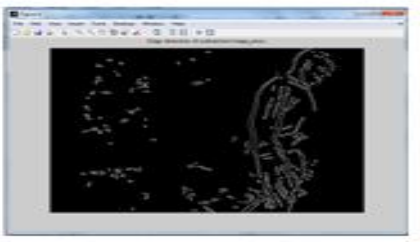

(e)

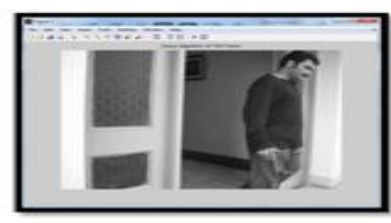

(b)

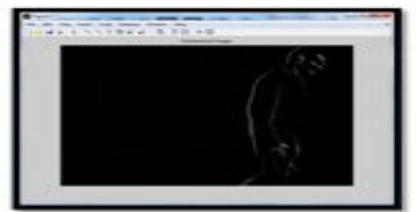

(d)

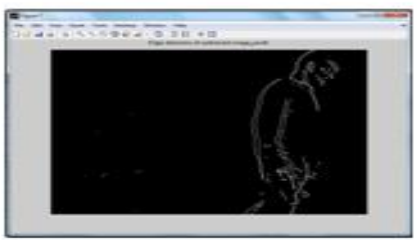

(f) 
Figure2. (a) Source Video (b) Captured frame-70 (c) Captured frame-71 (d) Difference image.
(e) Edge detection by Canny operator.
(f) Edge detection by Prewitt operator.

In this paper we have analyzed and made visual comparison between Canny and Prewitt edge detection operators. Visual comparison between fig (e) and fig (f) shown that prewitt is more suitable in case of motion tracking.

\section{V.II Motion Tracking: Background Elimination Using Single Prewitt Operator.}

Step1: Read Video Sequences by using Video Reader Object.

Step2: Capture two images and store it in IMG and IMG1. IMG is the static background image having no moving object. IMG1 is the $\mathrm{N}^{\text {th }}$ number of frame having moving object.

Step3: IMG: = Convert Frame to Gray Scale.

Step4: IMG1: = Convert Frame number $\mathrm{N}$ to Gray Scale.

Step5: Subtract frame IMG from frame IMG1, to generate the difference image.

Step6: Remove noise by using median filter.

Step7: Run Prewitt operator on the difference image in order to remove noise and to detect the edges.

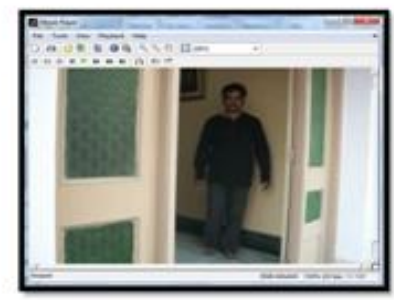

(a)

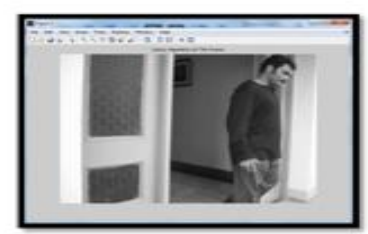

(c)

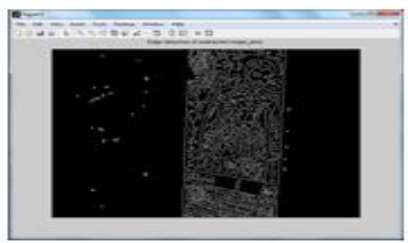

(e)

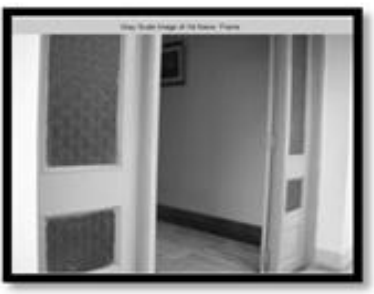

(b)

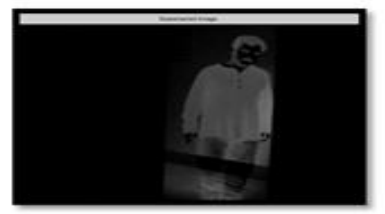

(d)

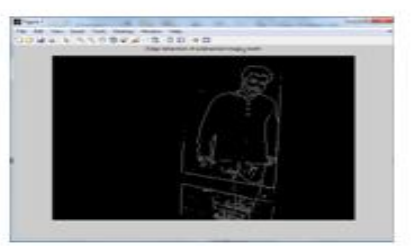

(f)

Figure3. (a) Source Video (b) Captured frame-45(c) Captured frame-71 (d) Difference image. (e) Edge detection by Canny operator. (f) Edge detection by Prewitt operator.

V.III Motion Tracking By Using Double Prewitt Operators.

Step1: Read Video Sequences by using Video Reader Object.

Step2: Capture two images and store it in IMG and IMG1.

Step3: IMG: $=$ Convert Frame number $\mathrm{N}$ to Gray Scale.

Step4: IMG1: $=$ Convert Frame number N +1 to Gray Scale.

Step5: EDGE: = Applying prewitt edge detection on IMG.

Step6: EDGE1:= Applying prewitt edge detection on IMG1.

Step7: Subtract EDGE from EDGE1, to generate the difference image. 


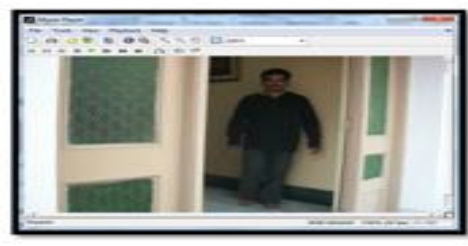

(a)

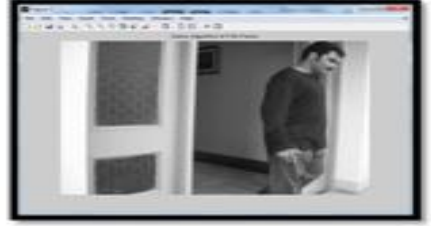

(b)

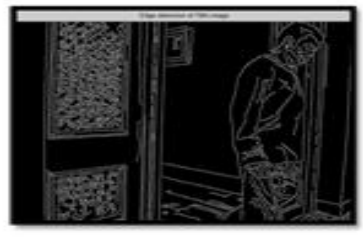

(d)

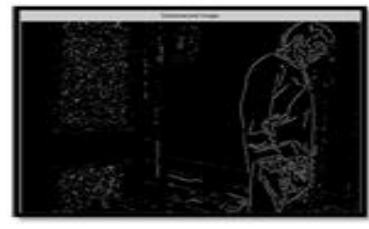

(f)

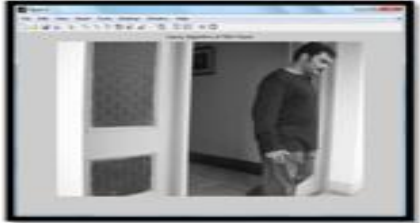

(c)

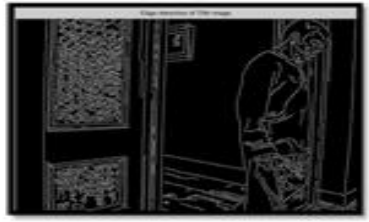

(e)

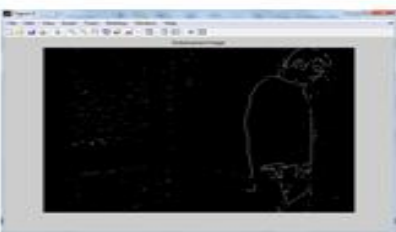

(g)

Figure4. (a) Source Video (b) Captured frame-71 (c) Captured frame-72 (d) Edge detection-71.

(e) Edge detection-72. (f) Difference image using Canny operator. (g) Difference image using Prewitt operator.

V.IV Motion Tracking By Using Single Prewitt Operator And Crop Function.

Step1: Read Video Sequences by using Video Reader Object.

Step2: Capture two images and store it in IMG and IMG1.

Step3: IMG: $=$ Convert Frame number $\mathrm{N}$ to Gray Scale.

Step4: IMG1: $=$ Convert Frame number $\mathrm{N}+1$ to Gray Scale.

Step5: Subtract frame IMG from frame IMG1, to generate the difference image.

Step6: Applying CROP function to Select the particular area of an image and store it into a variable (say Crop).

Step7: Run Canny filtering on the Crop image in order to remove noise and to detect the edges.

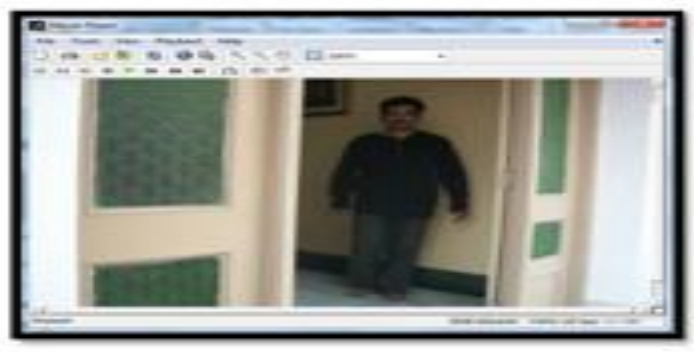

(a) 


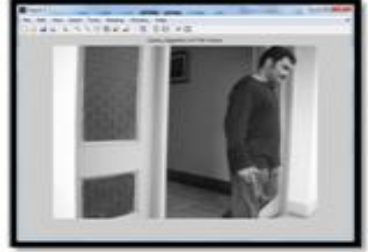

(b)

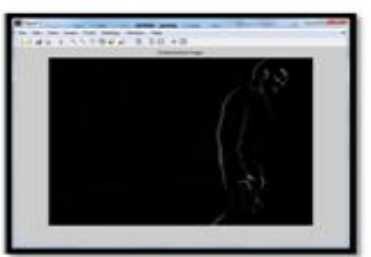

(d)

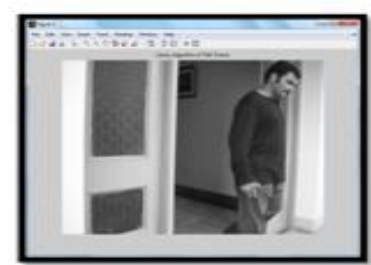

(c)

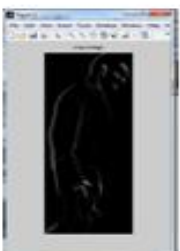

(e)

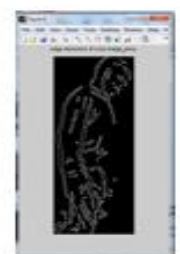

(f)

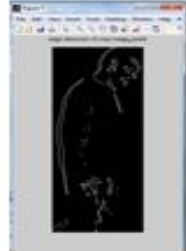

(g)

Figure5. (a) Source Video (b) Captured frame-71(c) Captured frame-72 (d) Difference image.(e) Croped image.(f) Edge detection using Canny operator. (g) Edge detection using Prewitt operator.

V.V Motion Tracking By Using Background Subtraction On Color Images And Applying A Single Prewitt Operator.

Step1: Read Video Sequences by using Video Reader Object.

Step2: Capture two consecutive colour images and store it in IMG and IMG1.

Step3: Subtract IMG from IMG1, to generate the difference image.

Step4: Convert the difference image to gray scale image.

Step5: Remove noise by using median filter.

Step6: Run prewitt operator on the Gray scale image in order to remove noise and to detect the edges.

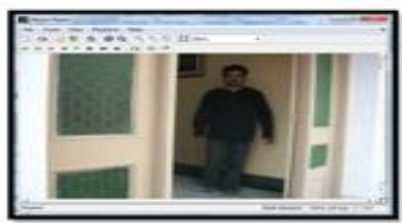

(a)
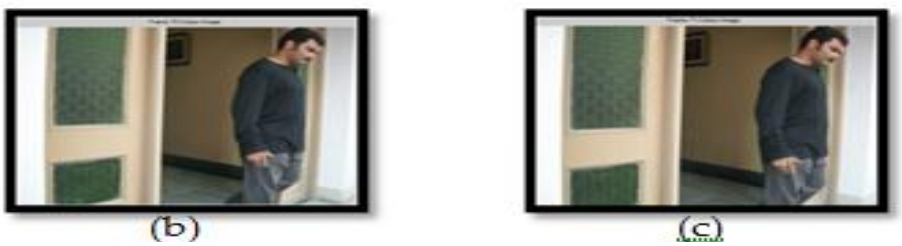

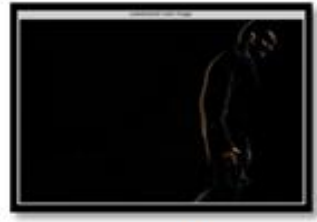

(d)

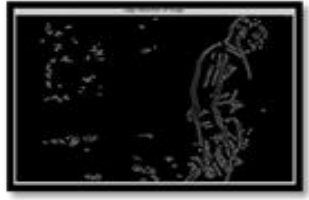

(f)

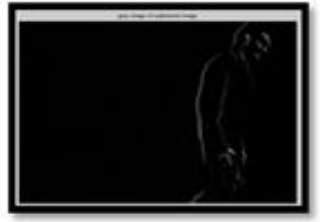

(e)

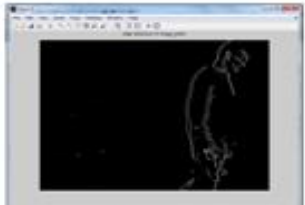

(g)

Figure6. (a) Source Video (b) Captured frame- 
71 (c) Captured frame-72 (d) Colour difference image. (e) Gray scale difference image. (f) Edge detection using Canny operator. (g) Edge detection using Prewitt operator.

\section{Conclusion}

In this paper we have proposed five different techniques of motion tracking by using background subtraction method and two edge detection operators: "Prewitt" and "Canny". By analysis and visual comparing the experimented result we come to conclusion that prewitt edge detection operator is more suitable than canny edge detection operator for motion tracking. As canny operator detects maximum number of edges, this leads to noise when we perform background subtraction method for tracking moving objects.

Acknowledgements

We tender our heartily gratitude to our parents, family members and friends.

\section{REFERENCES}

[1] Rafael C. Gonzalez, Richard E.Woods, Digital Image Processing, Prentice Hall

[2] J.Canny, “A Computational Approach to Edge Detection”, IEEE Trans.1986.

[3] Arnab Roy, Sanket Shinde and Kyoung-Don Kang, International Journal of Image Processing(IJIP), Voloume (2),

[4] A.Mukherjee, "Edge detection based motion tracking in video survellance", Proc. International Conference on Signal and Image Processing,2013.(Conference Proceedings)

[5] Merin Antony A,JAnitha, "A Survay of moving object segmentation methods".

[6] M.Piccardi,"Background subtraction technique: a review", IEEE Conference on System,Man and Cybernetics. 